2

3

4

5

6

7

8

9

10

11

12

13

14

15

16

17

18

19

\title{
A Brief History of Risk
}

\author{
Ying Li, ${ }^{\text {a }}$ Thomas Hills, ${ }^{\text {b Ralph Hertwig }}{ }^{\mathrm{a}}$
}

${ }^{a}$ Center for Adaptive Rationality, Max Planck Institute for Human Development, 14195

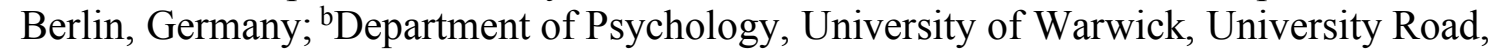
Coventry, CV4 7AL, United Kingdom

We thank Susannah Goss for editing the manuscript.

Corresponding Author: Ying Li

Contact: 1i@mpib-berlin.mpg.de 


\section{Abstract}

21 Despite increasing life expectancy, and high levels of welfare, health care and public safety in most post-industrial countries, the public discourse often revolves around perceived threats. Terrorism,

23 global pandemics, and environmental catastrophes are just a few of the risks that dominate media 24 coverage. Is this public discourse on risk disconnected from reality? To examine this issue, we analyzed the dynamics of the risk discourse in two natural language text corpora. Specifically, we tracked latent semantic patterns over a period of 150 years to address four questions: First, we examined how the frequency of the word risk has changed over historical time. Is the construct of risk playing an ever-increasing role in the public discourse, as the sociological notion of a 'risk society' suggests? Second, we investigated how the sentiments for the words co-occurring with risk have changed. Are the connotations of risk becoming increasingly ominous? Third, how has the meaning of risk changed relative to close associates such as danger and hazard? Is risk more subject to semantic change? Finally, we decompose the construct of risk into the specific topics with which it has been associated and track those topics over historical time. This brief history of the semantics of risk reveals new and surprising insights - a fourfold increase in frequency, increasingly negative sentiment, a semantic drift towards forecasting and prevention, and a shift away from war toward chronic disease - reflecting the conceptual evolution of risk in the archeological records of public discourse. 
A Brief History of the Semantics of Risk

\section{Introduction}

Humans have always been exposed to risks. Yet the nature of these risks has changed profoundly over the course of human biological and cultural evolution. Whereas the dominant risks were once starvation, infections, and violent conflict (Harari 2016), many of today's risks are associated with lifestyle choices (e.g., obesity, cardiovascular disease, cancer). Although modern institutions such as hospitals, police and fire services, and international treaties now buffer people in industrialized nations from the worst consequences of risks, the "consequences of modernity" (Giddens 1990) include new risks, such as nuclear weapons, global pandemics, deadly hospital bugs, terrorism, cyberattacks, and climate change. As we write this text, the world discusses and prepares for the coronavirus and has seen the worst fires' in Australia's recorded history. Despite reductions in the rates of violent conflict, poverty, and starvation (Pinker 2011) and a doubling of life expectancy over the past two centuries (Oeppen and Vaupel 2002), many people appear to feel that the world is more rife with dangers than ever (see Pinker 2011). Indeed, the historian Bourke (2005) has argued that "fear is the most pervasive emotion of modern society." Relatedly, life in today's "risk society” (Beck 1992) has been characterized as by rising vigilance to a growing variety of risks and insecurities (e.g., the precautionary principle; Sunstein 2005). Others have diagnosed a "current climate of fear" (Stearns, 2012, p. x), at least partly fueled by a range of players (e.g., politicians, media, federal agencies, businesses) who are desperate to capture public attention and are willing to inflame fear in the process. The conjecture that people are more afraid then they used to be is also a regular topos in the cultural discourse (Rothman, 2016). It is not clear, however, that people are have more fears today than they used to have because firm data on a population's fear level and risk perception only go back so far. In addition, answers to surveys are influenced by the current cultural context, and some have argued: "Currently, fear has become in some ways slightly fashionable, so maybe people are even exaggerating a little bit" (Stearns in Rothman, 2016).

How does society identify risks? Cultural anthropologists and sociologists have emphasized that risks are not a natural kind but are socially constructed, based on norms, moral considerations, and structures of social organization (Douglas 1992). What qualifies as a risk is therefore subject to dynamic social change. For instance, today's religiously motivated terrorism is a striking example of how an "old" risk transforms into a new phenomenon and forcefully reappears on the collective radar. Bourke (2005) has documented a history of fears, from the Victorians' dread of being buried alive to the more recent fear of nuclear annihilation. These fears are preserved in cultural artifacts such as books and newspaper articles-records that provide insights into how risks are collectively identified and perceived. Taking a historical perspective on these artifacts reveals how and why society's attitudes to risk have changed and may indicate how they will change again in the future. Our goal is to take a largescale quantitative approach to the recent historical trajectory of the word risk with the aim of understanding the changing nature of its social construction. 
Before we turn to our research questions, let us clarify that the term risk is often used to mean different things. In the risk management and actuarial literature, for instance, it describes a loss of a certain magnitude (e.g., injury, mortality) weighted by the probability of its occurrence (Short Jr, 1984; Rayner \& Cantor, 1987). By this actuarial measure, driving is riskier than flying because it is associated with a greater risk of injury per mile travelled. In the economic discourse, risk commonly refers to the variance in possible (positive or negative) returns. For instance, an investment option with higher return variance is deemed as riskier than an option with lower variance but the same expected mean return (Markowitz, 1952; Pratt 1964). Research in psychology, sociology, and anthropology has consistently demonstrated that these actuarial and economic definitions are too narrow to capture people's understanding of risk. Lay perceptions are multidimensional, encompassing higher order factors such as dread and equitable exposure (Slovic, 1987; Bhatia, 2019). Dread risks, as opposed to chronic risks, are defined by a perceived lack of control and potential large-scale loss of life, making flying a greater perceived risk than driving (e.g., Gaissmaier \& Gigerenzer 2012). Greater dread, in turn, is associated with greater perceived risk and a greater desire for regulation to reduce the risk (Slovic et al., 1985; Slovic, 1987; Sunstein 2005). All these meanings and others are part of the public discourse and are included in the text corpora that we analyze. In other words, our focus is not on one definition at the expense of another, but rather endorses the rich and inclusive semantic history of risk in the natural language.

\section{Guiding Research Questions}

Our goal in this article is to track change in the public discourse on risk over historical time by addressing four guiding questions. First, we examine how the frequency of the word risk has changed over historical time. Word frequency has been used to capture patterns of usage associated with changes in cultural importance (Twenge et al., 2012: Greenfield, 2013; Uz, 2014). Here, it allows us to evaluate the idea that the construct of risk is playing an ever-increasing role in the public discourse, suggested, for instance, by the sociological notion of a "risk society" (Beck 1992) as well as the anthropological observation of the word risk gaining large prominence (Douglas, 1992, p. 14). Second, we investigate how the sentiments for the words co-occurring with risk have changed. This sentiment analysis allows us to evaluate the hypothesis that risk is becoming a more negative construct associated with the expectation that societies and policy makers invest ever more in risk reduction and prevention (the precautionary principle; Sunstein 2005). Third, we ask how the meaning of risk has changed by

110 examining change in the semantic relationship between it and other words. The meaning of a word can 111 be reliably inferred from the contexts in which it has been used (Firth 1957). For example, analysis of 112 the linguistic context of the verb broadcast shows that 150 years ago it referred to the spreading of seed, 113 while it is now used to mean the spreading of information ( $\mathrm{Li}$ et al. 2019). We examine the text corpora 114 for indications that risk is more subject to semantic change than close semantic associates such as 115 danger and hazard. Risk, this seemingly neutral combination of chance and harm, has, so at least in the 
116 view social anthropologist Douglas (1992) a strong cultural foundation. This foundation is not static

117 but perspectives and social environments change, some dangers are politized as risks and other worries

118 are backgrounded. If risk has became a crucial construct to single out certain objective dangers and

119 designate them as social concerns, then underlying risk is dynamic mechanism constantly responding

120 to the changing sociocultural environment (Douglas, 1992). Fourth, we decompose the construct of risk

121 into the specific topics with which it has been associated and track those topics over historical time.

122 Our purpose here is to identify the most prominent risk topics over time and to consider how they have changed in relation to world events.

We investigated these questions by analyzing latent semantic patterns in natural language. Tracing the historical meanings of words requires a corpus of texts published over a sufficiently long time period. The Google Books Ngram Corpus (Lin et al., 2012) is one of the few corpora that meet this requirement. Drawing on over 100 sources (e.g., libraries and publishers), it contains over 8 million

128 books published from 1600 to 2008 , or $6 \%$ of all books ever published. The corpus thus offers a

129 telescopic view over a large time period. The corpus has been used to detect large-scale changes in 130 language, which in turn correlate with social and demographic changes (Michel et al., 2011; Hills et al. 131 2012; Hills \& Adelman, 2015, Hills et al., 2015). Any corpus, however, has its limitations. The Google 132 Books Ngram Corpus offers limited contextual information due to a narrow window size (5-grams, or 133 a contiguous sequence of five words); moreover, there has been a surge in the proportion of academic 134 articles in the corpus (Pechenick et al., 2015). We therefore also examined The New York Times 135 Annotated Corpus (NYT corpus; Sandhaus, 2008) to lend convergent validity to our results. This corpus 136 contains all (1.8 million) articles published in the New York Times from 1987 to 2007, and offers a more 137 microscopic view on the risks of modern life as reported in the most widely read U.S. newspaper. Let 138 us emphasize that because our analysis draws on English texts only, the present results are limited to 139 English-speaking cultures. In addition, the two corpora can of course provide only a limited window 140 onto the public discourse on risk. Nevertheless, the Google Books Ngram Corpus, in particular, has the 141 advantage of covering a relatively long time period, going beyond short-term analyses of, for instance, media coverage of risk and mortality (see the references in Young et al., 2008).

\section{$143 \quad$ 3. Materials and Methods}

144 In our analysis, we used word co-occurrence to construct semantic representations of risk in 145 each year so that the meaning of risk is approximated by the context in which it was used. The co146 occurrence information allows us to quantify how the sentiment and semantics of risk have changed 147 over history. As risk may be used in multiple contexts, we used Latent Dirichlet Allocation (LDA, Blei 148 et al. 2003) to identify the historical risk topics. This topic model algorithm detects underlying topics 149 that best explain the structure of the language around risk, and allowed us to identify risk topics as they 150 changed over time. In what follows, we describe this procedure in more detail. We begin by first briefly 151 describing the Corpora we used. 


\subsection{Google Books Ngram Corpus}

The Google Books Ngram Corpus consists of $n$-grams: contiguous sequences of $n$ items from

154 a given text ( $n$ ranges from 1-5). We used the 5-grams of all English words in our analysis; each data entry therefore displays the number of times a 5-gram appears in the corpus during a specific year. We retrieved all 5-grams starting or ending with the word risk. As is standard procedure in many natural language processing tasks, we removed stop words, punctuation, digits, and words containing fewer than three characters before using the WordNet-based NLTK lemmatizer (Bird et al., 2009) to lemmatize each noun to its singular form and each verb to its present tense. Next, we aggregated the corpus by year so that each document contains all 5-grams in a specific year. Aggregating topics by years encourages the topic model to identify the underlying patterns that best explain differences among risk structures over years.

\subsection{The New York Times Annotated Corpus}

The NYT Corpus contains all articles published in the New York Times from 1987 to 2007. We constructed a risk corpus by selecting articles that mentioned the word risk or risks more than twice. Next, we pre-processed the corpus in the same way as we did the Google Books Ngram data, apart from aggregating articles by year: Each news article was treated as one document.

\section{$168 \quad 3.3 \quad$ Corpus of Historical American English}

The Corpus of Historical American English (COHA) is a large structured corpus of historical English. It contains 400 million words of text from 1810s - 2000. COHA is balanced by genre decade by decade, which brings both benefits and concerns. On one hand, it alleviates concerns that insights learnt from the corpus are driven by the changing compositions of genres. However, on the other hand,

173 balanced genre may fail to map the reality that public preference of genres changes over history.

174 Although it is difficult to argue whether COHA is a better corpus for the analysis of culture change than the Google Ngram corpus or the other way around, it brings more convergent validity when findings from both corpora converge. Therefore, we used COHA to validate some of the historical analysis we did with the Google Ngram Corpus, namely analysis on frequency and semantic shift.

\section{$178 \quad 3.4 \quad$ Analysis of Frequency and Contextual Sentiment.}

179 Analyses of frequency, contextual sentiment, and semantic drift (Figures 1 and 2) were conducted using 180 the Macroscope ( $\mathrm{Li}$ et al. 2019), an interactive linguistic tool that analyzes historical sentiment and semantic change. The Macroscope was built on the basis of the historical word co-occurrence data made publicly available through the Google Books Ngram Corpus. Frequency was calculated by dividing the count of the selected words by the corpus size to control for the different corpus sizes for each year. Contextual sentiment for the selected words was computed in terms of the averaged valence ratings of co-occurring words during a given year. The valence ratings were retrieved from data collected by Warriner et al. (2013), which contain valence scores for 13,915 English words, each rated on its

187 "pleasantness" by around 30 participants. Using contemporary norms to estimate the valence of words 188 decades ago is challenging since all words may have changed their meaning or sentiment over history. 
189 However, in practice, historical sentiment inferred from averaging contemporary valence norms of 190 semantic neighbors has been found to be similar with the sentiment judged by historical language 191 experts (Buechel, Hellrich, \& Hahn, 2016).

$192 \quad 3.5 \quad$ Semantic Shift Analysis.

193 The purpose of semantic drift analysis is to examine how and to what extent the meaning of 194 risk has changed over the past two centuries in relation to related concepts such as fear, danger, and 195 hazard. Semantic drift analysis consisted of the following three steps: The first step is to retrieve 196 historical word embeddings trained by $\mathrm{Li}$ et al. (2019). Word embeddings provide a vector 197 representation for each word based on its co-occurring relationship with other words. Therefore, it 198 represents the context in which a word has been used. To derive the word embeddings, Li et al (2019) 199 first constructed a large co-occurrence matrix for 50,000 common English words that records number 200 of times any two words have been used within the same 5-gram. Next, they computed the positive pointwise mutual information (PPMI) for each pair of words and then constructed a PPMI matrix with entries given by:

$$
\operatorname{PPMI}\left(v_{i}, v_{j}\right)=\max \left(0, \log \left(\frac{p\left(v_{i}, v_{j}\right)}{p\left(v_{i}\right) \times\left(v_{j}\right)}\right)\right),
$$

where $v_{i}, v_{j}$ represents a pair of words from the corpus, and $\mathrm{P}(\mathrm{v})$ corresponds to the empirical probabilities of word co-occurrences within a sliding window of five over the original text. Finally, they reduce the dimension of word embeddings to 300 using singular value decomposition (SVD). This dimensionality reduction acts as a form of regularization and allows us to compare word similarities by computing the cosine similarity of word embeddings.

As the second step, based on historical word embeddings trained by Li et al. (2019), we identified the 20-nearest semantic neighbors for words risk, danger, hazard, and fear. Specifically, for each of the four target words, we retrieved word embeddings in year 1800 and year 2000. For risk, we also include its historical embeddings for every 20 years from 1800 and 2000. In order to compare word embeddings from different time-periods we must ensure that the vectors are aligned to the same coordinate axes. Therefore, we used orthogonal Procrustes to align the historical embeddings (Schönemann, 1966).

The third step was to visualize semantic shift of words in two-dimensional space. To this end, we used principal component analysis to reduce dimensions of word embeddings from 300 to 2 . The word embeddings retrieved in step 2 are plotted according to the two orthogonal principal components (PC1 and PC2 in figure 2). These two PCs represent compressed dimensions that best explains variance of the raw data and are therefore not directly interpretable except in relation to relative distance between word embeddings. The background words (semantic neighbors) are always shown in their "modern" (year 2000) positions. This approximation is necessary since, in reality, all words are moving. Risk and its synonyms are shown in their modern and historical positions. The path travelled through the semantic space is a proxy for change in historical meaning. 
Finally, to validate our observations, we quantified semantic change of risk and its related concepts using historical word embeddings trained on COHA (Hamilton et al, 2016) and on Google Ngram Corpus (Li et al, 2019). For each word, we computed cosine similarity between its embeddings trained on the $1820^{1}$ corpus and the 2000 corpus.

\subsection{Topic Modelling}

We studied historical change in the meaning of the word risk by extracting risk topics from the Google Books Ngram Corpus (Lin et al. 2012) and the NYT corpus (Sandhaus 2008). The topic model we used was Latent Dirichlet Allocation (LDA; Blei et al. 2003), a bag-of-words algorithm that identifies a set of topics that best describe/re-generate the corpus. We took two main steps in analyzing the data. First, we identified the structure of risk meanings by applying the topic model to the risk corpus. This step allowed us to understand the key events associated with risk. Next, we applied trend analysis to understand how the risk topics identified in the first step changed over time.

\subsection{Interpreting Topics}

To make sense of the meanings of the risk topics, we used Equation (1) to identify the words most relevant to each topic. The relevance of term $w$ to topic $k$ given a weight parameter $\lambda$ was defined as:

$$
\gamma(w, k \mid \lambda)=\lambda \log \left(P(w \mid k)+(1-\lambda) \log \left(\frac{P(w \mid k)}{P(w)}\right),\right.
$$

where $P(w \mid k)$ is the probability of term $w$ being assigned to topic $k$ and $P(w)$ is the marginal probability of term $w$ being in the corpus. The first component of the equation, $P(w \mid k)$, prioritizes terms with high frequency in a topic. However, it does not consider how unique term $w$ is to topic $k$, which can be captured by $\frac{P(w \mid k)}{P(w)}$, a quantity that Taddy (2012) called lift. We set $\lambda$ to 0.5 to take both components into consideration; $\lambda$ determines the weight given to the probability of term $w$ under topic $k$ relative to its lift.

One issue with topic models is that it is not clear which topics capture structures specific to the risk corpus and which topics capture general features of the source corpus. To find out, we used Equation (2) to compute the specificity of topic $k$ to the risk corpus:

$$
\operatorname{Specificity(k)}=\sum_{i=1}^{n}\left(\frac{\gamma\left(w_{i} \mid k\right)}{\sum_{i=1}^{n} \gamma\left(w_{i} \mid k\right)} * \frac{p\left(w_{i} \mid \text { risk corpus }\right)}{p\left(w_{i} \mid \text { general corpus }\right)}\right),
$$

252 where $\frac{\gamma\left(w_{i} \mid k\right)}{\sum_{i=1}^{n} \gamma\left(w_{i} \mid k\right)}$ is the normalized relevance of word $w$ to topic $k$, and $\frac{p\left(w_{i} \mid \text { risk corpus }\right)}{p\left(w_{i} \mid \text { general corpus }\right)}$ is the ratio of the frequency of word $w$ in the risk corpus to its frequency in the source corpus. Specificity can range from 0 to almost infinity. A specificity of 1 means that, on average, the words characterizing the topic have the same frequency in both the risk corpus and the source corpus, suggesting that the topic reflects

\footnotetext{
${ }^{1}$ We choose year 1820 instead of 1800 because frequency of risk in COHA before 1820 proved too small to train a stable model.
} 
256 the underlying pattern of the source corpus, not risk. An example of a nonspecific topic is one that 257 generates the words necessary to construct every document, such as articles and pronouns. The absolute 258 value of topic specificity is heavily influenced by the data format: NYT articles are more likely than 5259 grams to contain non-risk-specific words (noise) and therefore have smaller values of

$260 \frac{p\left(w_{i} \mid \text { risk corpus }\right)}{p\left(w_{i} \mid \text { general corpus }\right)}$. Topic specificity is not comparable across corpora; instead, it should be used 261 to compare topics from a same corpus.

\section{$262 \quad 3.8 \quad$ Tracking Trends in Topics}

263 To analyze trends in topics over time, we used the output from the LDA model on the Google Books 264 Ngram Corpus to calculate the contribution of each topic $k$ in each year by applying Equation (3). For 265 each document (i.e., all 5-grams in a specific year), the equation controls for document length by 266 dividing the number of words generated by each topic by the total number of words in the document. 267 Thus, the yearly topic contribution estimate, $p_{d}(k)$, is defined as:

$$
p_{d}(k)=\frac{|\{w \in d: \operatorname{topic}(w)=\mathrm{k}\}|}{|d|},
$$

269 where $k$ is a topic and $w$ is a word in a document $d$. The numerator is the number of words in document $d$ that are generated by topic $k$; the denominator is the total number of words in document $d$.

\section{4. $\quad$ Results}

\subsection{How Has the Frequency of Risk Changed Over Time?}

We first investigated change in the frequency of the word risk over time, starting with the

274 Google Books Ngram Corpus. As Figure 1A shows, use of the word risk has increased dramatically 275 since about 1970, with an approximately fourfold increase in usage since the 1950s. We checked this 276 trend in English against other languages and found similar increases in French, German, Italian, and 277 Spanish (Figure 1B). In addition, we observed a similar proliferation of risk in the Corpus of Historical 278 American English (COHA; Davies 2008). As COHA is balanced by genre and subgenre across 279 decades, $^{2}$ these findings suggest that risk proliferation is not an artifact of increasing numbers of 280 scientific journals being included in the Google Books Ngram Corpus (Figure 1A). There is, however, no sign that the public discourse has turned darker in general, as close semantic relatives signifying undesirable states such as fear, danger, and hazard are not being used more frequently. On the contrary, the use of fear and danger has declined steadily over the past two centuries, while the use of hazard has remained relatively stable at a low frequency. These results are consistent with the idea that risk, more than other terms, has become a central concept in recent and present public and political discourses (Beck, 1992; Bourke, 2005; Douglas, 1992).

\footnotetext{
${ }^{2}$ For example, fi ction accounts for $48-55 \%$ of the total in each decade (1810s-2000s); subgenres such as prose, poetry, and drama are likewise balanced. This balance across genres and subgenres means that researchers can be reasonably certain that patterns in the data do not merely reflect artefacts of a changing genre balance.
} 

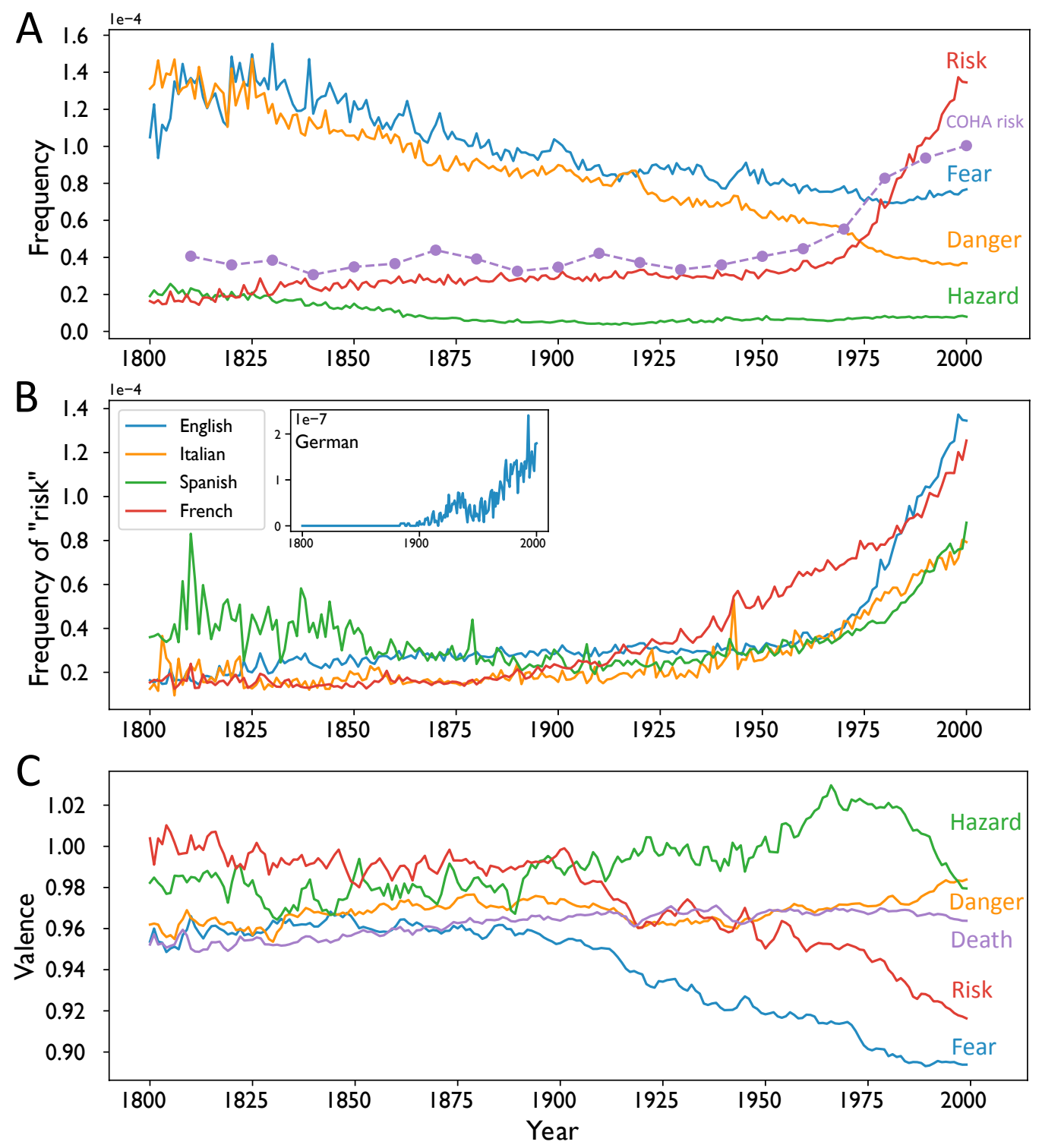

Figure 1. Historical change in the frequency and sentiment for the word risk and its close semantic neighbors in the Google Books Ngram Corpus. (A) Frequency of risk, fear, danger, and hazard in the Google Books Ngram Corpus and frequency of risk in the Corpus of Historical American English (COHA). (B) Frequency of risk in five languages-English, Italian, Spanish, French, and German - in the Google Books Ngram Corpus. German is presented in a separate box because the frequency of risk in German is much less than in other languages. (C). Change in the sentiment for words co-occurring with risk, fear, danger, hazard, and death. Sentiment was adjusted to mean score of all words, such that valences $>1$ indicate a more positive context than average. The word death is included to provide a sentiment benchmark, as its meaning and sentiment have remained stable over history.

\subsection{How Have the Sentiments Associated with Risk Changed?}

Next, we examined whether the sentiments ${ }^{3}$ associated with risk have changed over time. For example, is it possible - in line with a more economic interpretation of risk - that the use of the word

\footnotetext{
${ }^{3}$ We found it not necessary to distinguish between sentiment of the word and sentiment of the context in which the word was used. Since we inferred historical sentiment by averaging the valence of contextual neighbors, what we measure is sentiment of the context associated with risk, not directly sentiment of the meaning of risk.
} 
301 risk is increasingly associated with an appreciation of the large potential rewards that make some risks

302 worth taking (Pleskac \& Hertwig, 2014)? This is not the case, as the results presented in Figure 1C

303 show. Computing the frequency-weighted average valence of the words that co-occurred with risk over

304 the past 200 years revealed that the sentiment associated with risk has become increasingly negative,

305 showing a roughly monotonic decline from 1800 to 2000 . To provide points of comparison, we also

306 analyzed the related concepts of fear, danger, hazard as well as death as benchmarks. The sentiment

307 analysis shows that risk has undergone a much larger change over time than these inherently undesirable

308 concepts (with the exception of fear). In the early 1800s, the sentiment for words co-occurring with risk

309 was more positive than that of any of the four comparison words; by the end of $20^{\text {th }}$ century, it was more

310 negative than that of danger, hazard, or death (Figure 1C). In other words, the word risk has become

311 not only more prevalent but also more negative in meaning.

312

\section{$313 \quad 4.3 \quad$ How Have the Semantic Relationships of Risk Changed?}

314 The increasing negativity of risk's sentiment, relative to the stability of the sentiment for risk,

315 fear, danger, and hazard, might be driven by the changing contexts in which these words have been

316 used. Therefore, in this section we turn to an analysis of semantic drift, which likewise suggests that

317 the semantics of risk have experienced more change over historical time than its close semantic

318 associates. Specifically, Figure 2 visualizes the semantic associates of risk, danger, hazard, and fear in

319 two-dimensional space relative to their $k$ most similar words in 1800 and 2000 ( $k=9$ for each word).

320 Larger distance between two words suggests less similarity in the contexts in which they appeared. The

321 pattern is clear: risk, danger, and hazard started as close semantic neighbors in 1800 and moved apart

322 over time. By the year 2000, the underlying semantics of risk had grown more similar to those of

323 prevalence and prevention, terms associated with the quantification, reduction, and avoidance of risk.

324 Danger and hazard, in contrast, remained in the semantic area defined by words such as harm, threat,

325 adverse, and peril. This finding suggests that the word risk has moved from merely representing the

326 presence of threats, to also being associated with the scientific examination, quantification, and

327 prevention of threats.

328 It is possible that this pattern is a result of an increase in the amount of academic (especially

329 medical) articles in the Google Ngram corpus (Pechenick et al. 2015). Therefore, we again used COHA,

330 a smaller yet genre-balanced corpus, to validate our findings. We analyze the semantic shift of risk

331 using historical word embeddings trained on COHA (Hamilton et al, 2016) and compared the results

332 with results derived from on embeddings trained on Google Ngram Corpus (Li et al., 2019). For each

333 word, we quantify semantic similarity of a word over history by computing cosine similarity between

However, these two are conceptually related: a word used in negative context is likely to carry negative connotations since meaning of word can be learnt from the linguistic companions it keeps (Firth, 1957). 
334 its embeddings trained on $1820^{4}$ corpus and 2000 corpus. Cosine similarity score ranges from 0 to 1

335 with larger scores suggesting greater semantic similarity. Comparison of analysis on the two corpora 336 confirms that the semantics of risk is much less stable than danger, hazard, and fear (Table 1). In 337 addition, we searched for the nearest semantic neighbor for risk in COHA in year 1820 and year 2000.

338 Again, we find that risk acquires associations with medical concepts: its top-5 nearest semantic 339 neighbors derived from COHA change from loss, expense, danger, trouble, run, in year 1820 to disease, 340 diabetes, cancer, rate, and factors in year 2000.

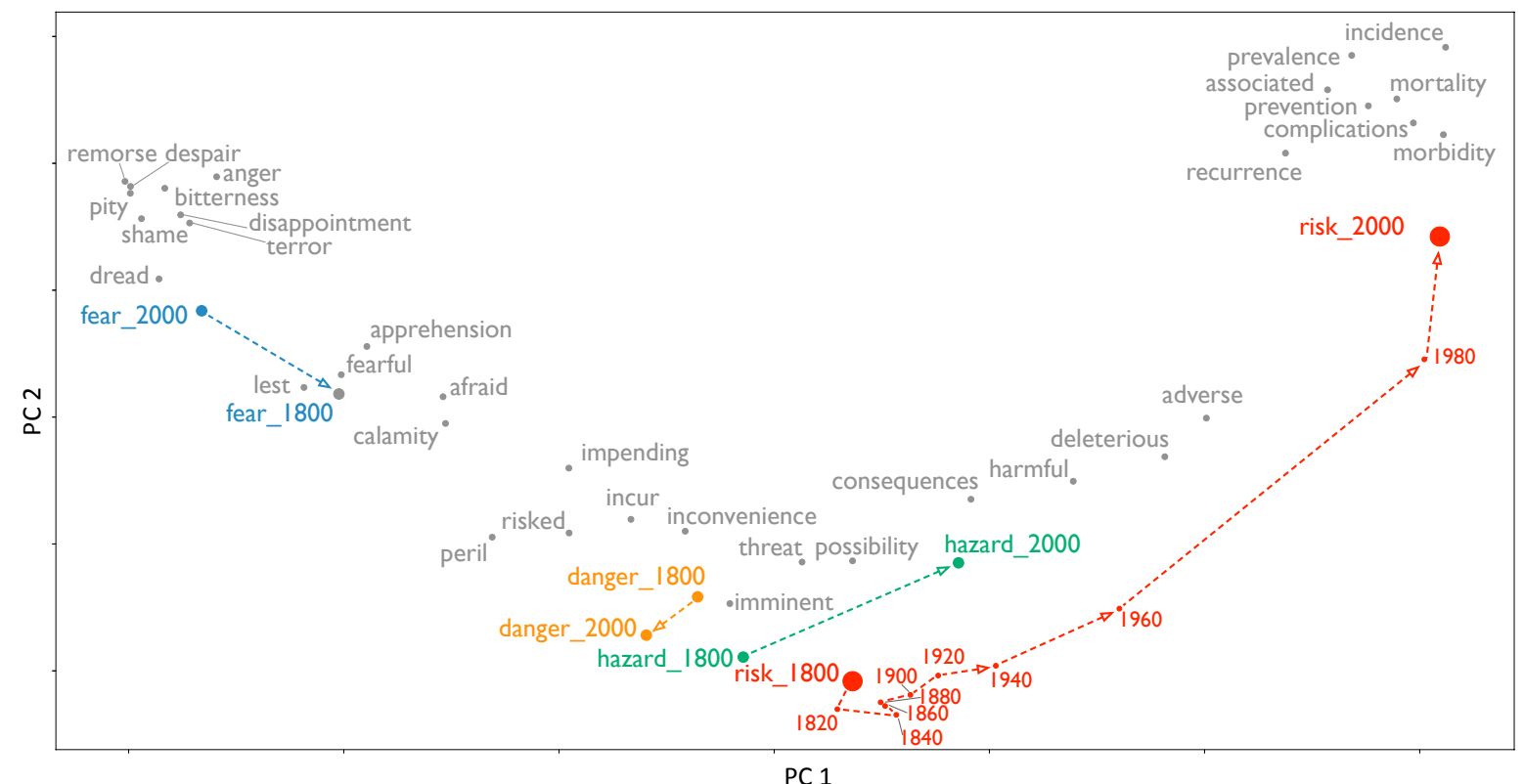

341

342

343

344

345

346

347

348

349

Figure 2. Semantic drift of risk, hazard, danger, and fear from 1800 to 2000 in the Google Books Ngram Corpus. The target words (risk as red dots; the other three as green dots) are shown in relation to their near associates (as blue dots) in the years 1800 and 2000. PCA was performed to reduce the dimension of word embeddings from 300 to 2 so that words can be visualized in two-dimensional space. The axes represent the two principal components. Larger distance between two words suggests less semantic similarity. The words risk, danger, and hazard started as near neighbors in 1800 but moved apart over time.

Table 1. Semantic similarity between year 1820 and 2000.

\begin{tabular}{lllll}
\hline & Risk & Danger & Fear & Hazard \\
\cline { 2 - 5 } Google Ngram & 0.36 & 0.61 & 0.58 & 0.56 \\
COHA & 0.42 & 0.81 & 0.80 & 0.54 \\
\hline
\end{tabular}

350 Note: For each word, semantic similarity was inferred by taking cosine similarity between word 351 embeddings of year 1820 and 2000. The embeddings were normalized so that the similarity scores range 352 from 0 to 1 , with 1 and 0 representing maximum and minimum similarity, respectively.

\subsection{How Have Risk Topics Changed Over Time?}

\footnotetext{
${ }^{4}$ We choose year 1820 instead of 1800 because frequency of risk in COHA before 1820 is too small to train a stable model.
} 
The semantic drift analysis shows how risk has diverged from its semantic neighbors over the last two centuries, but it fails to provide details on the topical dimensionality of risk in this period. As noted by Blais and Weber (2006), risk is a multidimensional concept encompassing numerous topics. We therefore applied LDA to investigate the topics that have driven the proliferation of risk in the public discourse and its increasingly negative sentiment. We inferred topic meanings by inspecting their most relevant words (see Equation 1 in the Methods section), as summarized for each topic in Table 2. Applying the topic model to the Google Books Ngram Corpus identified six risk categories: war (topic 1, 2, 3), nuclear (topic 4), health (topic 5, 6, 7, 8, 9), HIV/AIDS (topic 10, 11), risk society (topic 12), economy (topic 13,14), and a non-specific topic on risk analysis (topic 15).

Table 2. Most Relevant Words for Each Risk Topic, Ordered by Relevance as Defined in Equation 1

\begin{tabular}{|c|c|c|c|}
\hline Index & Google Books Ngram Corpus & Index & NYT Corpus \\
\hline 1 & Life, imminent, battle, resolve & 1 & Military, war, Iraq, troop \\
\hline 2 & Life, war, bureau, loss & 2 & China, Japan, country, foreign \\
\hline 3 & War, uncertainty, loss, prepare & & \\
\hline 4 & Nuclear, carcinogenic, patient, infant & 3 & Environmental, plant, energy, gas \\
\hline 5 & Heart, coronary, injury, bear & 4 & Cancer, woman, study, breast \\
\hline 6 & Breast, cancer, osteoporosis, fetus & 5 & Drug, patient, doctor, hospital \\
\hline 7 & Stroke, cancer, disease, capital & & \\
\hline 8 & Prostate, cancer, event, Alzheimer & & \\
\hline 9 & $\begin{array}{l}\text { Management, diabetes, cardiovascular, } \\
\text { overweight }\end{array}$ & & \\
\hline 10 & AIDS, nation, HIV, immunodeficiency & 6 & AIDS, virus, infect, vaccine \\
\hline 11 & HIV, deficit, assess, volume & & \\
\hline 12 & Management, value, assessment, society & 7 & Child, school, parent, student \\
\hline 13 & Confrontation, return, equilibrium, preference & 8 & Fund, stock, investor, market \\
\hline 14 & Rate, free, interest, return & & \\
\hline \multirow[t]{13}{*}{15} & Behavio[u]r, group, death, population & & \\
\hline & & 9 & Food, fat, eat, diet \\
\hline & & 10 & Insurance, bank, loan, insurer \\
\hline & & 11 & Law, court, abortion, tobacco \\
\hline & & 12 & Airline, flight, shuttle, space \\
\hline & & 13 & Company, business, executive, industry \\
\hline & & 14 & Investigation, Enron, prison, police \\
\hline & & 15 & Think, people, way, thing \\
\hline & & 16 & Republican, Clinton, Bush, Democrat \\
\hline & & 17 & Game, player, sport, team \\
\hline & & 18 & Day, car, hour, walk \\
\hline & & 19 & City, build, York, new \\
\hline & & 20 & Film, art, movie, theater \\
\hline
\end{tabular}

Note: Topic 15-20 of NYT corpus are shown in light grey to indicate that these topics are not specific to articles that contains the word risk and its inflections. Topic specificity is defined in equation 2.

Each topic represents a probability distribution over all words. In order to validate our interpretation of risk topics from the Google Books Ngram Corpus, we selected a collection of words (see the left column of Figure 3A) that characterize each of the risk categories identified above and examined how those words were distributed over topics (see the left panel of Figure 3A). To provide 
372 further validation of our interpretation of topics, instead of selecting words from table 2, we chose a 373 different set of associates that we felt exemplify our interpretation of the topics based on corresponding 374 events at the time of the topics peak. For example, under the war category, we selected words that reflect 375 the major war participants in 20th century such as Soviet, American, Japan, Germany, as well as war376 related words such as battle, invasion, and war etc. For the cancer category, we include names of the 377 most common cancer. If our interpretation was correct, topics that we grouped under the same category 378 should be more likely to generate corresponding words but not others. This is what we find. For example, 379 Figure 3A shows that topic 1, 2 and 3 in the Ngram corpus (identified as war topics in table 2) associate 380 with the set of words we selected under the war category, such as war, world, invasion, army, battle, 381 etc. This pattern, visualized as probability loadings on the diagonal of the word-topic probability heat map in Figure 3A, supports the interpretation of topic meanings in Table 2.

How replicable is this category structure? To find out, we also analyzed the NYT Corpus. 384 Applying the same procedure to the NYT Corpus confirmed all risk categories inferred for the Google 385 Books Ngram Corpus (visualized as probability loadings on the diagonal of the right panel of Figure 386 3A). We can therefore conclude that the meanings of risk derived in our analysis of the Google Books Ngram dataset are not corpus-specific results associated with a non-representative sample, but reflect general trends in the topicality of risk over both relatively long and short time scales.

In order to ensure that the topics were risk-specific and did not just reflect the background features of the corpus, we next computed topic specificity (see Equation 2 in the Methods section) to quantify the relative correspondence of each topic with the risk corpus as compared with the entire corpus (see Figure 3B). A topic specificity score around or below 1 means that the topic has a distribution of words similar to that seen in the entire corpus; the topic therefore represents the general features of the entire corpus. For the Google Books Ngram Corpus, we found the topic specificity of all risk topics to be above 1 (ranging from 50 to 650), suggesting that all topics were risk-relevant. In contrast, the specificity of NYT topics ranged from 0.7 to 2.5 , with six topics being irrelevant to risk (the specificity scores of topics 15-20 were close to or less than 1). This notable difference in the topic specificity of the two corpora may be attributable to differences in data format: Recall that the Google Books Ngram data contain words that co-occurred with risk within a narrow window size, whereas the

400 NYT data contain entire articles that mention the word risk. As such, NYT articles are more likely than Google Books Ngrams to contain words not specific to risk.

Nevertheless, both corpora rendered a similar set of high-specificity topics: nuclear, heart disease, cancer, diabetes, and HIV/AIDS. War-related topics had low specificity in the NYT Corpus.

404 This result is not surprising because, as we show in the following analysis, war topics have gradually disassociated from risk since World War II, and the NYT Corpus only dates back to 1987. Beyond the risk topics identified for the Google Books Ngrams, we found only one additional topic in the NYT

407 Corpus with specificity clearly above 1 (topic 9, featuring words such as food, fat, eat, and diet), and 408 four additional NYT topics slightly above 1 (topics 11-14, which we interpreted as legal, flight, 
commercial, and fraud, respectively). Correspondingly, the key words associated with topics 11-14

410 showed low co-occurrence with risk in the Google Books Ngram Corpus throughout history. This

411 comparison suggests that, overall, both corpora converged on a similar set of important risk categories.

412

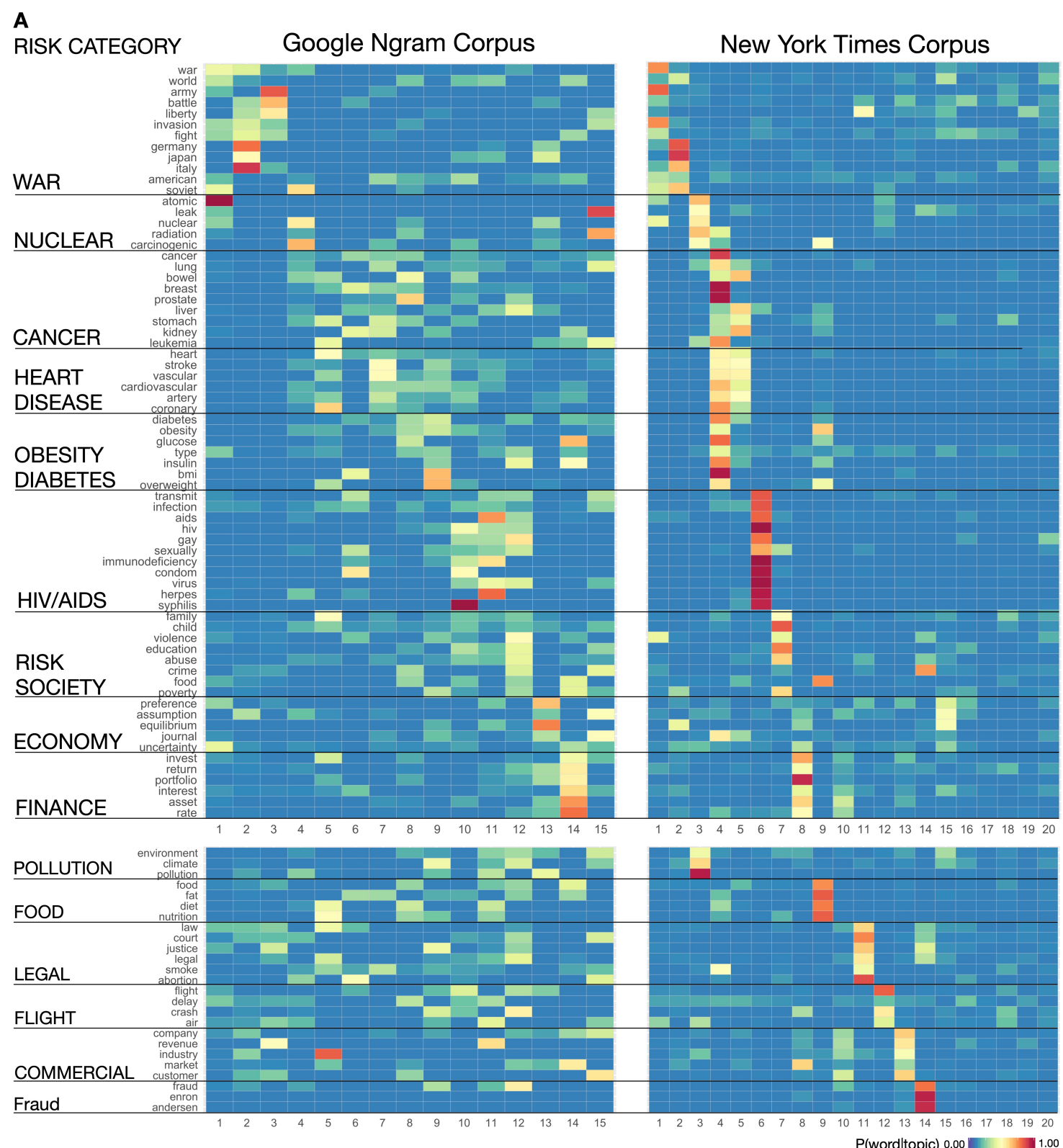

B
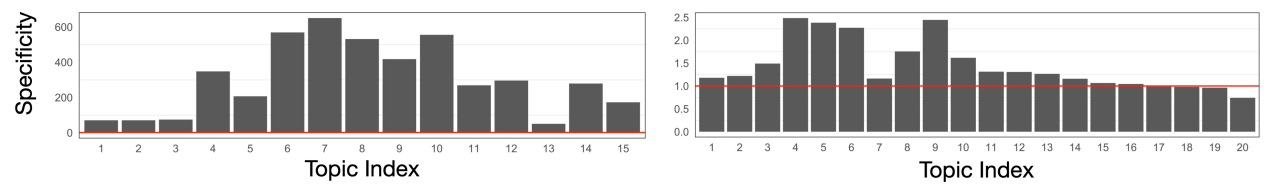

413

414

415

416

417

418

419

Figure 3. Visual quantification of risk topics. (A) Heatmap of the probability that word $w$ was generated by topic $k$ in models derived from the Google Books Ngram Corpus (left) and the NYT Corpus (right). Words on the $y$-axis were selected by referring to the list of most relevant words for each topic (relevance defined by Equation 1) and they were grouped by categories. (B) Topic specificity (as defined by Equation 2). The red horizontal line indicates topic specificity equal to 1 . Topics with specificity above this reference line can be considered risk-specific and therefore capture one or more 
420

421

422

423

424

425

426

427

428

429

430

431

432

433

434

435

436

437

438

439

440

441

442

443

444

445

446

447

448

449

450

451

452

453

454

aspects of the meaning of risk. Topics with specificity below 1 can be considered generic words that are not informative with respect to risk meanings.

\subsection{How Are Changes in Risk Categories Associated with Other Events and Developments?}

One advantage of Google Books Ngram Corpus is that it allows us to investigate change in the sources of risk across a period of over 150 years and to speculate on how those changes relate to other historical events and developments. Specifically, we performed a trend analysis on the topic model derived from the Google Books Ngram Corpus over the years 1850 to 2008. As Figure 4 shows, the structure of the Google Books Ngram risk topics underwent major changes over this period. The three war-related topics emerge early in the distribution: Topic 1 (life, imminent, battle, resolve) dominated the risk structure in the second half of the $19^{\text {th }}$ century, which witnessed several major wars (e.g., Crimean War, American Civil War). Topic 2 (life, war, bureau, loss) emerged and reached its peak during World Wars I and II. Topic 3 (war, uncertainty, loss, prepare) reached its peak during the Vietnam War. Topic 4 (nuclear, carcinogenic, patient, infant) peaked around 1985, capturing the risks associated with the proliferation of nuclear weapons during the Cold War (see the histogram in Figure 4) and the growing use of nuclear power in the 1970s and 1980s.

Chronic diseases such as heart disease and cancer are now the leading global risks for mortality (World Health Organization 2009). Topics reflecting this development (topics 5-9) started to emerge from the 1970s and remain the most prominent risk topics. Due to the large proportion of shared words associated with the different health conditions, topics $5,6,7$, and 8 show considerable overlap, that is, they share words that describe cancer, heart and coronary issues, and other severe diseases. Topic 9 , associated with obesity and diabetes, emerged after 2000. The data for topics 10 and 11 show that concerns over AIDS and HIV emerged within 2 years of the first AIDS diagnosis in the US in 1981 and soon reached a peak around 1995, when the reported annual mortality from HIV/AIDS peaked in the United States (CDC 2010). Potentially reflecting the dramatic medical advances in treatments for HIV and drop in mortality rates, this risk topic decreased in prominence after 2000 (see the histogram of AIDS-related deaths in the US in Figure 4).

Finally, topic 12 (management, value, assessment, society) is about management of various social risks. It seems to relate to Beck's conceptualization of the risk society, being associated with words such as Ulrich, Beck, and modernity. Topics 13 and 14 relate to the economy, and emerged from the 1970s: topic 13 features words like preference, assumption, equilibrium, and journal, whereas topic 14 features words such as return, portfolio, and interest. Lastly, topic 15 (behavior, group, death, population) seems to be concerned with general risk analysis, without reference to any specific risk event. 
Topic Trends: $1850-2008$
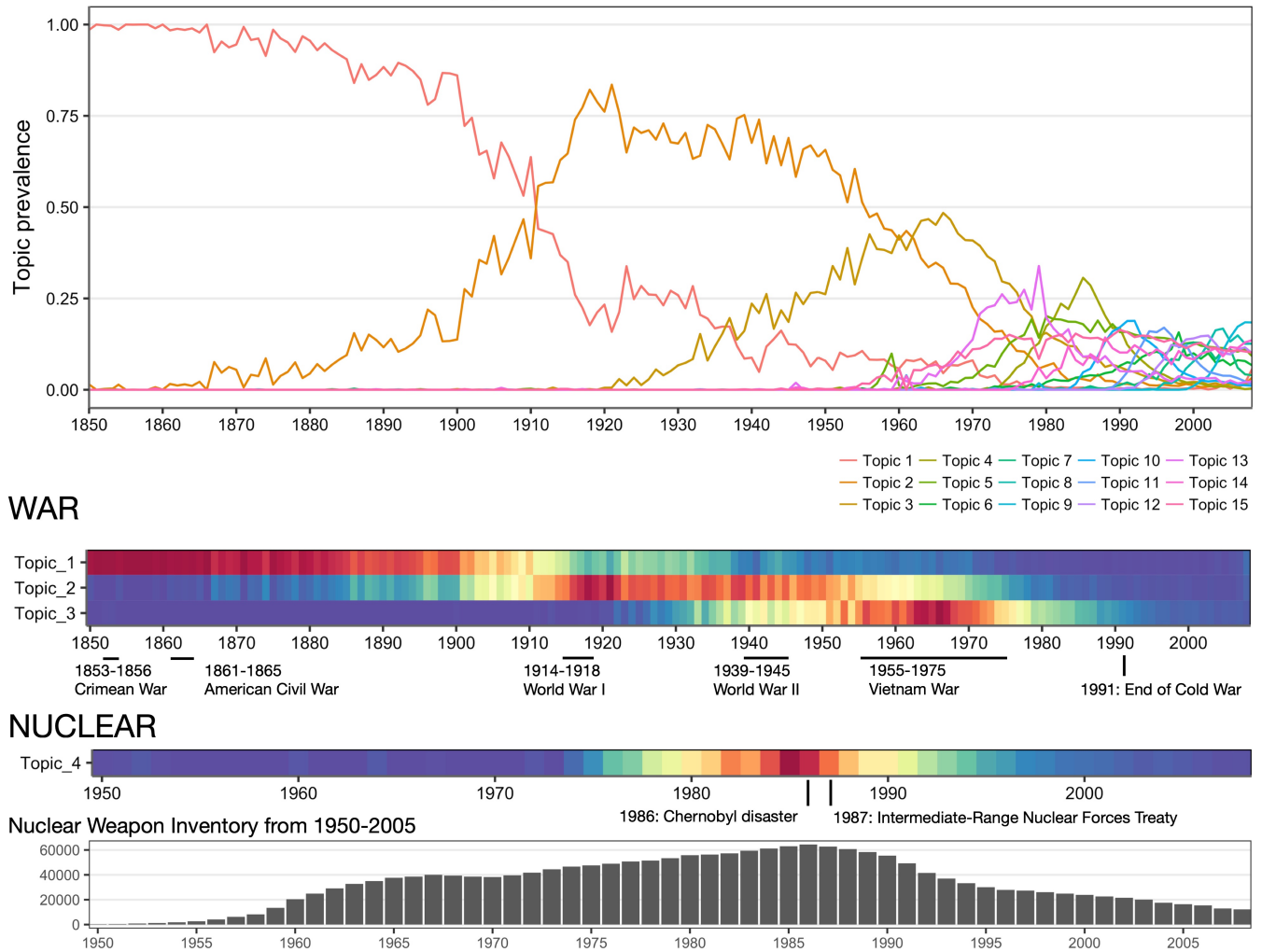

HEALTH: Heart disease, cancer, obesity and others.

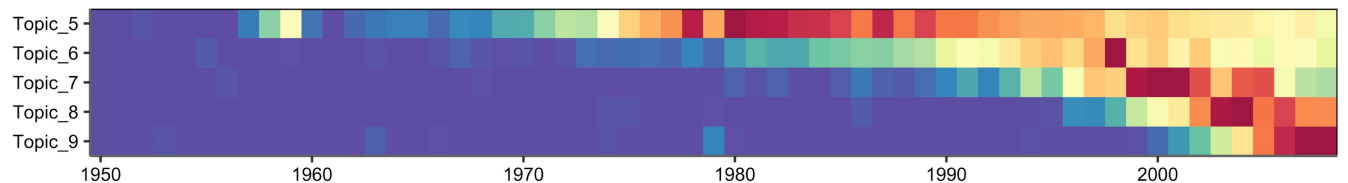

HIV/AIDS

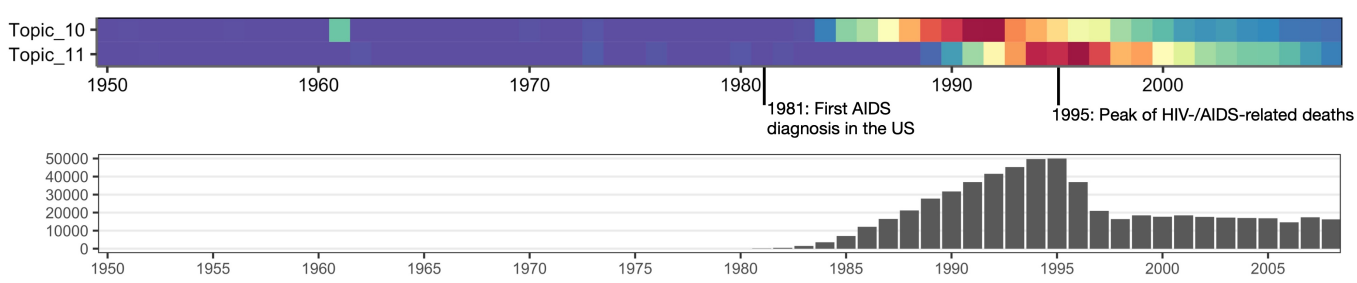

RISK SOCIETY
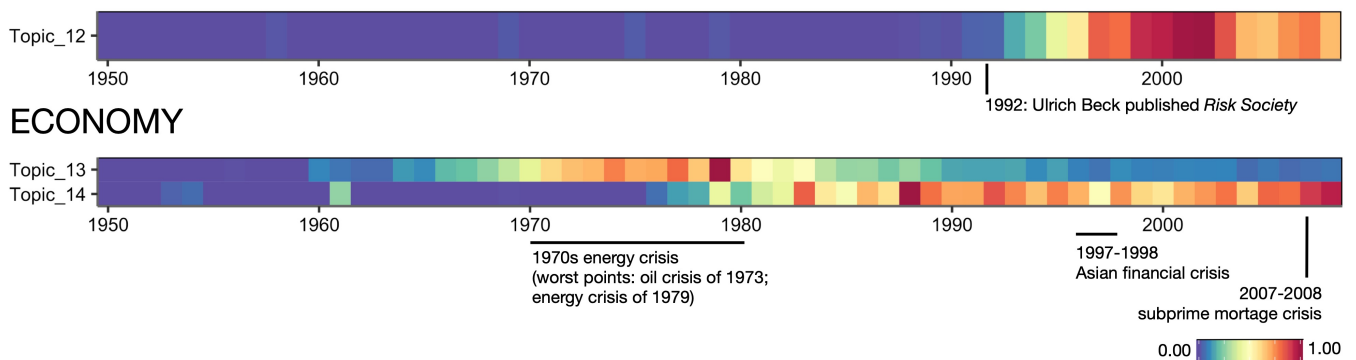

Figure 4. Trend analysis on risk topics derived from the Google Books Ngram Corpus. Topics are grouped into six categories: war, nuclear, health, HIV/AIDS, risk society, and economy. Relevant historical events are labeled to indicate how changes in the meanings of risk were associated with historical events and developments. Top panel: historical trends of 15 risk topics (computed using Equation 3). Bottom panel: normalized topic trend for each individual topic. Topic 15 is not included as it does not refer to a specific risk topic. 


\section{Discussion}

Risk is a complex multidimensional construct. It takes a variety of forms in public discourse and has, accordingly, been investigated in various ways. Each approach focuses on some aspects of the discourse at the expense of others. One common approach has been to analyze media coverage of risk as a leading source of information for the general public and experts alike (see, e.g., Coombs \& Slovic, 1979, and various references in Young et al., 2008). Our approach consisted in a large-scale analysis of historical text corpora. Such corpora are attractive because they collate a vast array of perspectives on an extensive historical time window: in the case of the Google Book Ngrams Corpus, over 8 million books and 150 years. What did we learn about the risk-related discourse in English-speaking countries?

First, we found - consistent with Beck's (1992) diagnosis of post-industrialist Western societies as risk societies facing a wide variety of unique and human-made risks and with Gidden's (1990) idea that society is increasingly preoccupied with the future and its safety - that the word risk has become much more prevalent (Figure 1A). There is evidence of an approximately fourfold increase in its usage since the 1950s. Beck also stressed that risks in the post-modern world are increasingly unknowable and unpredictable due to scientific and technological innovations having unanticipated consequences. It is possible that this process has contributed to our second major observation, namely, that the sentiments associated with risk have become much more negative, starting around 1900 and confirming Pinker's (2011) observation that humans have become increasingly preoccupied with the negative aspects of risk. Interestingly, the same does not apply to its close semantic relatives (Figure 1C). What is also puzzling is that this change in sentiments is happening at a time when the semantics of risk have become increasingly associated with notions of quantification, reduction, and preventionfindings that also challenge the idea that the increase in negative sentiments has been caused by the unknowability of risks. In addition, we found that the risk categories to some extent reflect real-world changes in the prevalence and magnitude of the respective risks (see Figure 4 and our analyses of nuclear proliferation and AIDS-related deaths). Finally, we also found a shift from macro-risks, such as war and battle, to more individual-specific, chronic risks such as disease (Holzmann \& Jørgenson, 2000) as well as shift toward more variability in risk topics. The strong focus on modern diseases suggests that the public discourse appears generally oriented towards the most prevalent sources of death and harm. This is noteworthy as several authors have advanced the argument that people are afraid of the wrong things (see Glassner, 2018; Renn, 2014; Schröder, 2018).

Many of these patterns observed are remarkable in part because they are monotonic: the notable increase in the frequency and negativity of the risk construct, and the increase in number of topics it encompasses. These changes are perhaps related to one another. One potential underlying mechanism is the social amplification of risk (Kasperson et al., 1988; Moussaid et al., 2015; Jagiello \& Hills, 2018): as information is transferred from one individual to another, people tend to share the more negative aspects of a risk at the expense of potential gains. In Jagiello and Hills (2018), an individual exposed to 
499 was communicated from one individual to the next, the focus shifted increasingly to the downsides of 500 nuclear power and away from its benefits. This pattern is consistent with the substantial evidence that 501 negative information has more influence on decision making than positive information (Ito et al., 1998;

502 Baumeister et al., 2001; Rozin \& Royzman, 2001). A second, related factor is that this effect may be 503 further amplified by increasing communication over the period of our analysis. As Herbert Simon (1971) 504 noted, "a wealth of information creates a poverty of attention" (pp. 40-41). With the unprecedented 505 amounts of information now available, all other things being equal, the absolute amount of negative 506 information has increased. In this environment, information that is better at being received, remembered, 507 and reproduced has a selective advantage (Hills, 2019). Though this mechanism applies to all 508 information, it may be especially true of prominent risks, which may self-reinforce their negativity more 509 rapidly via enhanced social communication (Jagiello \& Hills, 2018).

$510 \quad$ Based on our results, what may one conclude about the state of the public discourse on risk? 511 First and foremost, our analysis can offer only a glimpse of this complex and multi-dimensional 512 construct. Yet, we found results that were both disconcerting and reassuring. Primarily, the increasing 513 prevalence of the word risk is an indicator of its growing significance, which is in itself a double-edged 514 sword. Classifying something as a potential risk is likely to burden it with negative sentiments. Yet, 515 branding something a risk also appears to imply the chance of changing our fortune in relation to it. 516 Importantly, the text corpus analyses suggest that risk categories track real threats over the $20^{\text {th }}$ and $21^{\text {st }}$ 517 century, shifting from violent death to the modern world's chronic diseases and major risks of morbidity 518 and mortality. In this sense, the risk discourse is not at all divorced from changes in threats and changes 519 in the potential to mitigate them. 


\section{Reference}

Baumeister RF, Bratslavsky E, Finkenauer C, Vohs KD (2001) Bad is stronger than good. Review Gen. Psych. 5(4):323-370.

Beck U (1992) Risk Society: Towards a New Modernity (Sage, London, UK).

Bhatia S (2019) Predicting risk perception: New insights from data science. Management Sci., ePub ahead of print April 11, https://doi.org/10.1287/mnsc.2018.3121

Bird S, Klein E, Loper E (2009) Natural Language Processing with Python: Analyzing Text With the Natural Language Toolkit (O'Reilly Media, Newton, MA).

Blais AR, Weber EU (2006) A domain-specific risk-taking (DOSPERT) scale for adult populations. Judgm. Decis. Mak. 1(1):33-47.

Blei DM, Ng AY, Jordan MI (2003) Latent Dirichlet allocation. J. Mach. Learn. Res. 3(Jan):9931022.

Bourke J (2005) Fear: A Cultural History (Virago, London, UK).

Buechel, S., Hellrich, J., \& Hahn, U. (2016, December). Feelings from the Past-Adapting Affective Lexicons for Historical Emotion Analysis. In Proceedings of the Workshop on Language Technology Resources and Tools for Digital Humanities (LT4DH) (pp. 54-61)

Centers for Disease Control and Prevention (2010) HIV/AIDS Surveillance Report. Atlanta, GA: CDC.

Combs B, Slovic P (1979). Newspaper coverage of causes of death. Journalism Quart., 56(4), 837849.

Davies, M (2008). The corpus of contemporary American English: 450 million words, 1990-present. Accessed April, 2017, http://corpus.byu.edu/coca/

Douglas M (1992) Risk and Blame: Essays in Cultural Theory (Routledge, Abingdon, UK).

Firth, JR (1957). Papers in Linguistics 1934-1951 (Oxford University Press, London, UK).

Gaissmaier W, Gigerenzer G (2012) 9/11, Act II: A fine-grained analysis of regional variations in traffic fatalities in the aftermath of the terrorist attacks. Psychol. Sci. 23(12):1449-1454.

Giddens A (2013) The Consequences of Modernity (John Wiley \& Sons, New Jersey).

Glassner, B. (2018). The culture of fear: Why Americans are afraid of the wrong things. New York: Basic Books.

Greenfield PM (2013) The changing psychology of culture from 1800 through 2000. Psychol. Sci. 24(9):1722-1731.

Hamilton, W. L., Leskovec, J., \& Jurafsky, D. (2016). Diachronic word embeddings reveal statistical laws of semantic change. arXiv preprint arXiv:1605.09096.

Harari YN (2016) Homo Deus: A Brief History of Tomorrow (Random House, New York).

Hills, TT (2019). The dark side of information proliferation. Perspect. Psychol. Sci., 14(3):323-330. Hills TT, Adelman JS (2015) Recent evolution of learnability in American English from 1800 to 2000. Cognition 143:87-92. 
560

Hills TT, Jones MN, Todd PM (2012) Optimal foraging in semantic memory. Psych Rev 119(2):431440.

Hills TT, Proto E, Sgroi D (2015) Historical analysis of national subjective wellbeing using millions of digitized books. IZA Discussion Paper No. 9195.

Holzmann R, Jørgenson S (2000) Social risk management: A new conceptual framework for social protection, and beyond. Int. Tax Public. Finan. 8(4):529-556.

Hornik, K., \& Grün, B. (2011). Topic models: An R package for fitting topic models. Journal of Statistical Software, 40(13), 1-30.

Ito TA, Larsen JT, Smith NK, Cacioppo JT (1998) Negative information weighs more heavily on the brain: The negativity bias in evaluative categorizations. J Pers Soc Psych 75(4):887-900.

Jagiello RD, Hills, TT (2018) Bad news has wings: Dread risk mediates social amplification in risk communication. Risk Anal. 38(10), 2193-2207.

Kasperson RE, Renn O, Slovic P, Brown H, Emel J, Goble R, Kasperson JX, Ratick S (1988) The social amplification of risk: A conceptual framework. Risk Anal. 8(2):177-187.

Li, Y, Engelthaler, T, Siew, CS, Hills, TT (2019). The Macroscope: A tool for examining the historical structure of language. Behav. Res. Methods, ePub ahead of print February 11, https://doi.org/10.3758/s13428-018-1177-6

Lin Y, Michel JB, Aiden EL, Orwant J, Brockman W, Petrov S (2012) Syntactic annotations for the Google Books Ngram Corpus. Proceedings of the ACL 2012 System Demonstrations (pp 169-174). Association for Computational Linguistics.

Markowitz H (1952) Portfolio selection. J. Financ. 7(1):77-91.

Michel JB, Shen YK, Aiden AP, Veres A, Gray MK, Pickett JP, Hoiberg D, Clancy D, Norvig P, Orwant J, Pinker S (2011) Quantitative analysis of culture using millions of digitized books. Science 331(6014):176-182.

Moussaïd M, Brighton H, Gaissmaier W (2015) The amplification of risk in experimental diffusion chains. Proc. Natl. Acad. Sci. 112(18):5631-5636.

Oeppen J, Vaupel JW (2002) Broken limits to life expectancy. Science (296):1029-1031.

Pechenick EA, Danforth CM, Dodds PS (2015) Characterizing the Google Books corpus: Strong limits to inferences of socio-cultural and linguistic evolution. PLOS ONE, 10(10):e0137041.

Pinker S (2011) The Better Angels of Nature: The Decline of Violence in History and its Causes (Penguin, London, UK).

Pleskac TJ, Hertwig R (2014) Ecologically rational choice and the structure of the environment. $J$. Exp. Psychol. 143(5):2000-2019.

Pratt JW (1964) Risk aversion in the small and in the large. Econometrica (32):122-136.

Rayner S, Cantor R (1987) How fair is safe enough? The cultural approach to societal technology choice. Risk Anal. 7(1):3-9. 
Renn, O (2014) Das Risikoparadox: Warum wir uns vor dem Falschen fürchten (Fischer Taschenbuchverlag, Frankfurt am Main, Germany).

Rozin P, Royzman, EB (2001) Negativity bias, negativity dominance, and contagion. Pers. Soc. Psychol. Rev. 5(4):296-320.

Sandhaus E (2008) The New York Times Annotated Corpus. (Linguistic Data Consortium, Philadelphia).

Short Jr JF (1984) The social fabric at risk: Toward the social transformation of risk analysis. Am. Sociol. Rev. 49(6):711-725.

Schönemann, P. H. (1966). A generalized solution of the orthogonal procrustes problem. Psychometrika, 31(1), 1-10.

Schröder, M (2018). Warum es uns noch nie so gut ging und wir trotzdem ständig von Krisen reden (Benevento, Salzburg, Austria).

Simon H (1991) Designing organisations for an information-rich word. Greenberger M, ed. Computers, Communications, and the Public Interest (The Johns Hopkins Press, Baltimore, MA), 40-41.

Slovic P (1987) Perception of risk. Science 236(4799):280-285.

Slovic P, Fischhoff B, Lichtenstein S (1985) Characterizing perceived risk. Kates RW, Hohenemser C, Kasperson JX, eds. Perilous Progress: Managing the Hazards of Technology (Westview, Boulder, CO), 91-125.

Stearns, P. N. (2012). American fear: The causes and consequences of high anxiety. Routledge.

Sunstein CR (2005) Laws of Fear: Beyond the Precautionary Principle (Vol. 6) (Cambridge University Press, UK).

Taddy M (2012) On estimation and selection for topic models. Proceedings of the Fifteenth International Conference on Artificial Intelligence and Statistics (pp 1184-1193).

Twenge JM, Campbell WK, Gentile B (2012) Increases in individualistic words and phrases in American books, 1960-2008. PLOS ONE 7(7):e40181.

Uz I (2014) Individualism and first person pronoun use in written texts across languages. J. Cross. Cult. Psychol. 45(10):1671-1678.

Warriner AB, Kuperman V, Brysbaert M (2013) Norms of valence, arousal, and dominance for 13,915 English lemmas. Behav. Res. Methods 45(4):1191-1207.

Why Americans are more afraid than they used to be (2016, January 6), TIME. Retrieved from https://time.com/4158007/american-fear-history/.

World Health Organization (2009) Global Health Risks: Mortality and Burden of Disease Attributable to Selected Major Risks. (World Health Organization, Geneva).

Young, ME, Norman, GR, Humphreys, KR (2008). Medicine in the popular press: The influence of the media on perceptions of disease. PLoS One 3(10), e3552. 